\title{
Downregulation of fatty acid synthase complex suppresses cell migration by targeting phospho-AKT in bladder cancer
}

\author{
SHUAI-SHUAI ZHENG, JIAN-GANG GAO, ZHI-JUN LIU, XIN-HONG ZHANG, SHUAI WU, \\ BO-WEN WENG, YOU-LIN WANG, SI-CHUAN HOU and BO JIANG \\ Department of Urology, Qingdao Municipal Hospital, School of Medicine, \\ Qingdao University, Qingdao, Shandong 266071, P.R. China
}

Received January 30, 2015; Accepted November 19, 2015

DOI: $10.3892 / \mathrm{mmr} .2015 .4746$

\begin{abstract}
The aim of the present study was to investigate the effect of fatty acid synthase complex (FASN) on the migration capacity of bladder transitional cell carcinoma (BTCC) cells and the involvement of matrix metalloproteinase-9 (MMP-9) via targeting of phospho-AKT (p-AKT). FASN-specific small-interfering RNA (FASN-siRNA) was used to inhibit FASN gene expression in the 5637 and 253J BTCC cell lines. The knockdown efficiency of FAM-conjugated FASN-siRNA was confirmed by fluorescence microscopy. The migratory abilities of BTCC cells were assessed using a Transwell assay. Furthermore, protein and mRNA expression of FASN, p-AKT, AKT, and migration-associated protein MMP-9 were detected by western blot analysis. Treatment with FASN inhibitor Cer and FASN-siRNA decreased the migratory capacity of bladder cancer cells and reduced the levels of p-AKT as well as the expression of MMP-9. These results indicated that FASN inhibition suppressed the migratory capacity of BTCC cells through suppressing AKT activation and consequently reducing MMP-9 expression. Targeting FASN may represent a promising novel therapeutic strategy for BTCC.
\end{abstract}

\section{Introduction}

As the second most common type of genitourinary malignancy along with urothelial cancer, bladder cancer comprises $90 \%$ of all primary bladder cancers (1). In spite of the advancements in chemotherapy and surgical techniques for this cancer type, $\sim 50 \%$ of bladder cancer patients develop metastases and succumb to the disease (2). As targeted agents have had limited success in treating metastatic bladder cancer (3-5), the development of treatments with an enhanced ability to reduce

Correspondence to: Dr Bo Jiang, Department of Urology, Qingdao Municipal Hospital, School of Medicine, Qingdao University, 5 Donghaizhong Road, Qingdao, Shandong 266071, P.R. China E-mail: jiangboqdy@126.com

Key words: fatty acid synthase complex, cell migration, bladder cancer, matrix metalloproteinase-9, small interfering RNA the migratory capacity of bladder transitional cell carcinoma (BTCC) it is urgently required.

fatty acid synthase complex (FASN), which was discovered in 1994 by Kuhajda et al (6), is the only human protein with the ability to catalyze a reductive de novo synthesis of long-chain fatty acids from acetyl-coenzyme A (CoA) and malonyl-CoA using nicotinamide adenine dinucleotide phosphate (6). In most normal tissue types, except for adipose and liver tissues, FASN expression is low $(6,7)$. In addition, FASN is highly expressed in most types of human carcinoma and its inhibition may therefore hold promise as a strategy for cancer chemoprevention (8). Of note, selective inhibition of FASN has been used for lung cancer treatment (9). Thus, current research on the discovery of novel diagnostic tools and treatment methods for cancer focuses on fatty acid metabolism and the role of FASN in cancer (10).

A previous study by our group has reported high levels of FASN expression in BTCC, and revealed that inhibition of FASN suppressed phosphorylated AKT (p-AKT) and induced apoptosis in bladder cancer (11). However, to the best of our knowledge, the possible role of FASN in the migratory capacity of BTCC cells has not yet been assessed. The present study therefore examined the effects of FASN-specific small-interfering RNA (FASN-siRNA) and FASN inhibitor cerulenin (Cer) on BTCC-cell migration. Furthermore, the effects of FASN knockdown on the AKT pathway and the expression of matrix metalloproteinase (MMP)-9 were investigated in order to assess the molecular mechanism of the role of FASN in BTCC-cell migration. The present study revealed the implication of FASN in the migratory capacity of BTCC cells as well as an underlying mechanism, and indicated that targeting of FASN may represent a novel therapeutic strategy for BTCC.

\section{Materials and methods}

Cell culture and reagents. The 5637 and 253J bladder transitional cell carcinoma lines were purchased from the cell bank of the Chinese Academy of Sciences (Shanghai, China). The cells were cultured at $37^{\circ} \mathrm{C}$ in a humidified atmosphere containing 5\% $\mathrm{CO}_{2}$ in RPMI 1640 medium (Gibco; Thermo Fisher Scientific, Inc., Waltham, MA, USA) supplemented with $10 \%$ fetal bovine serum (Gibco; Thermo Fisher Scientific, 
A
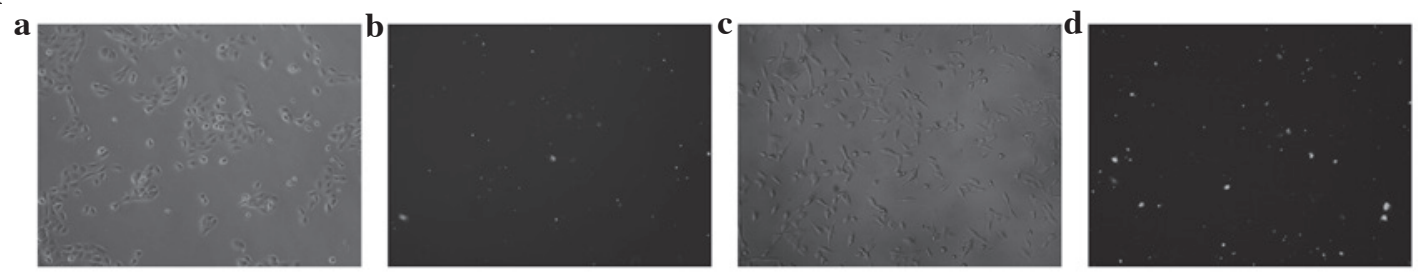

B
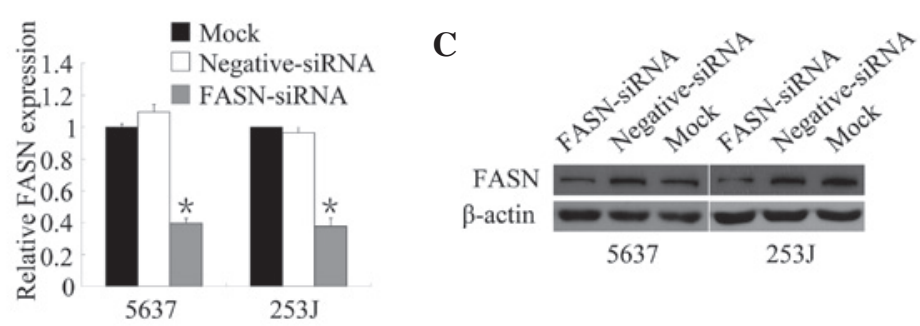

Figure 1. Transfection of 5637 and 253J bladder cancer cells with FASN siRNA. (A) 5637 cells were transfected with FAM-labeled negative siRNA for $12 \mathrm{~h}$ and the number of transfected cells was observed by (a) light microscopy and (b) fluorescence microscopy (magnification, x100). 253J cells were transfected with FAM-labeled negative siRNA for $12 \mathrm{~h}$ and the number of transfected cells was observed by (c) light microscopy (magnification, $\mathrm{x} 100)$ and (d) fluorescence microscopy (magnification, x100). Cells were mock-transfected or transfected with negative siRNA or FASN-siRNA for $12 \mathrm{~h}$, and the FASN knockdown efficiency was detected by (B) reverse transcription-quantitative polymerase chain reaction and (C) western blot analysis. Images are representative of three separate experiments. Values are expressed as the mean \pm standard deviation. ${ }^{*} \mathrm{P}<0.05$ vs. Mock and Negative-siRNA groups. FASN, fatty acid synthase complex; siRNA, small interfering RNA.

Inc.), $100 \mathrm{IU} / \mathrm{ml}$ penicillin and $100 \mu \mathrm{g} / \mathrm{ml}$ streptomycin (Gibco; Thermo Fisher Scientific, Inc.). Cells in the logarithmic growth phase were used in all experiments. Cer was obtained from Biomol (Enzo Life Sciences, Farmingdale, NY, USA) and kept in a $10 \mathrm{mg} / \mathrm{ml}$ stock solution in dimethyl sulfoxide (Gibco; Thermo Fisher Scientific, Inc.).

SiRNA and siRNA transfection. Using the nucleotide sequence of the FASN gene from GenBank (http://www.ncbi.nlm. nih.gov/genbank/; no. $004104 \mathrm{NM}$ ), siRNA to target FASN was designed (5'-CCCAGGCUGAAGUUUACA ATT-3'). Furthermore, a negative control siRNA sequence (5'-UUC UCCGAACGUGUCACGUTT-3'), named as Negative-siRNA, was designed. All siRNAs were designed and synthesized by Shanghai GenePharma Co., Ltd. (Shanghai, China). The 5637 and 253J cells were plated in six-well plates $(2 \mathrm{ml}$ medium/well) to reach $\sim 70 \%$ confluency at the time-point of transfection. Cells were transfected with FASN-siRNA in the experimental group, and with Negative-siRNA in the negative control group using Lipofectamine 2000 (Invitrogen; Thermo Fisher Scientific, Inc.) according to the manufacturer's instructions. After $12 \mathrm{~h}$ of transfection, cells were cultured in RPMI 1640 medium. To assess the transfection efficiency, FAM-siRNA (Shanghai GenePharma Co., Ltd.) was analyzed by fluorescence microscopy (BX50; Olympus Corporation, Tokyo, Japan).

Reverse transcription-quantitative polymerase chain reaction. Total RNA from cells were prepared using TRIzol reagent (Invitrogen; Thermo Fisher Scientific, Inc.). RNA was reverse-transcribed using an AMV Reverse Transcription System (Promega Corporation, Madison, WI, USA) and was amplified RT-qPCR. The sequences of the primers were as follows: FASN (sense, 5'-TATGCTTCTTCGTGC AGCAGTT-3'; antisense, 5'-GCTGCCACACGCTCCTCT AG-3'). The $\mathrm{Ct}$ values were normalized to the reference gene $\beta$-actin (sense, 5'-CGGGAAATCGTGCGTGA-3'; antisense, 5'-TGCCCAGGAAGGAAGGCT-3') and amplification was performed using SYBR Green as the fluorescent dye (Takara Biotechnology Co., Ltd., Dalian, China). The specificity of the PCR products was assessed with melting-curve analysis. The relative expression of mRNA was calculated using the comparative delta $\Delta \Delta$ Cq. (quantification cycle) method (12) to compare the expression levels among different samples.

Western blot analysis. Following transfection with FASN-siRNA for $48 \mathrm{~h}$ or treatment with Cer $(5$ or $10 \mu \mathrm{g} / \mathrm{ml})$ for $24 \mathrm{~h}$ in six-well plates, cells were harvested and re-suspended in $60 \mu 1$ lysis buffer (Gibco; Thermo Fisher Scientific, Inc.). Following determination of the protein concentration via the Bicinchoninic Acid Protein kit (Beyotime Institute of Biotechnology, Inc.), 30- $\mu$ g aliquots were separated by $8 \%$ sodium dodecyl sulfate-polyacrylamide gel electrophoresis (Gibco; Thermo Fisher Scientific, Inc.), followed by electrotransfer at $100 \mathrm{~V}$ onto a polyvinylidene fluoride membrane (Roche, Basel, Switzerland). Subsequent to blocking with 5\% non-fat milk in Tris-buffered saline containing $0.1 \%$ Tween 20 (TBST; Gibco; Thermo Fisher Scientific, Inc.) for $2 \mathrm{~h}$ at room temperature, the membrane was incubated with the following rabbit monoclonal primary antibodies at $4^{\circ} \mathrm{C}$ overnight: Anti-FASN (cat. no. 3189; 1:1,000), total-AKT (cat. no. 4691; 1:1,000), p-AKT (cat. no. 4060; 1:1,000), MMP-9 (cat. no. 3852; 1:1,000) or $\beta$-actin (cat. no. 4970; 1:1,000); Cell Signaling Technology, Danvers, MA, USA). Following three washes in TBST for $10 \mathrm{~min}$ each, the blots were incubated with horseradish peroxidase-conjugated anti-rabbit immunoglobulin G at 1:2,000 dilution (cat. no. 7074; Cell Signaling Technology) for $2 \mathrm{~h}$ at room temperature. Antigen was detected using standard chemical luminescence methodology. Immune complexes were visualized using an enhanced chemiluminescence kit (EMD Millipore, Billerica, MA, USA) and exposure to Biomax film (Kodak, Rochester, NY, USA). 
A
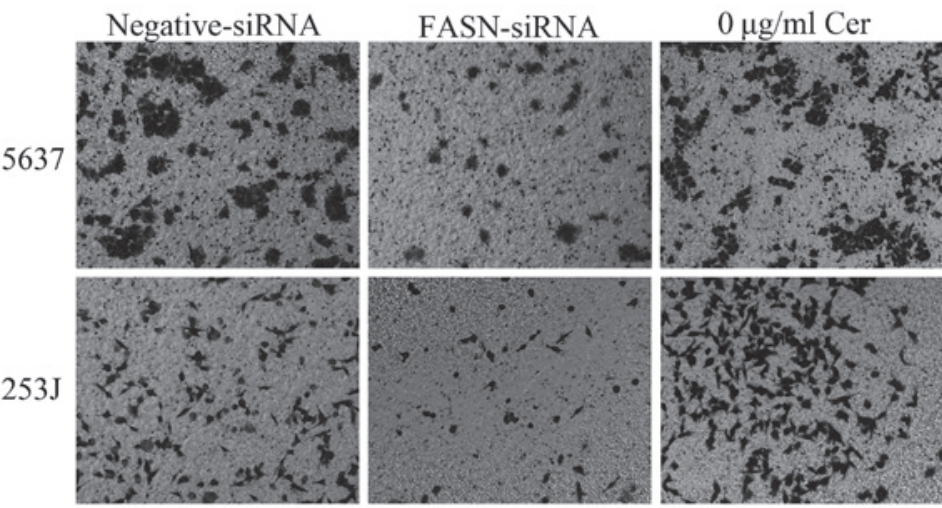

$3 \mu \mathrm{g} / \mathrm{ml} \mathrm{Cer}$
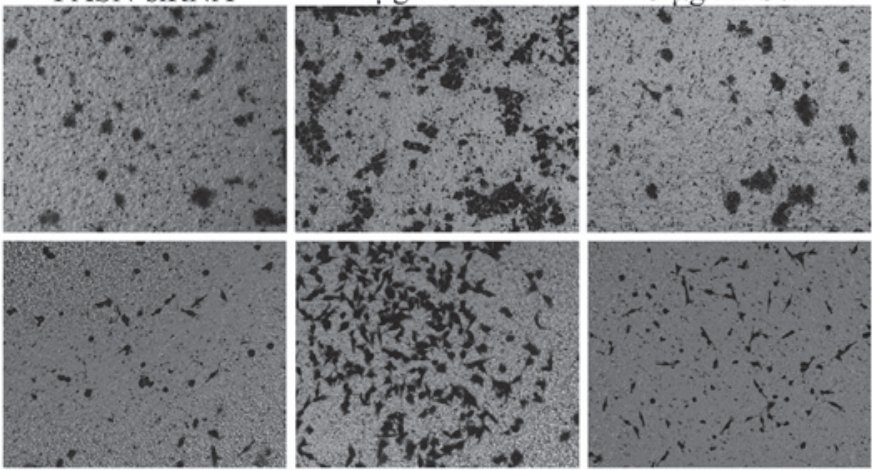

B

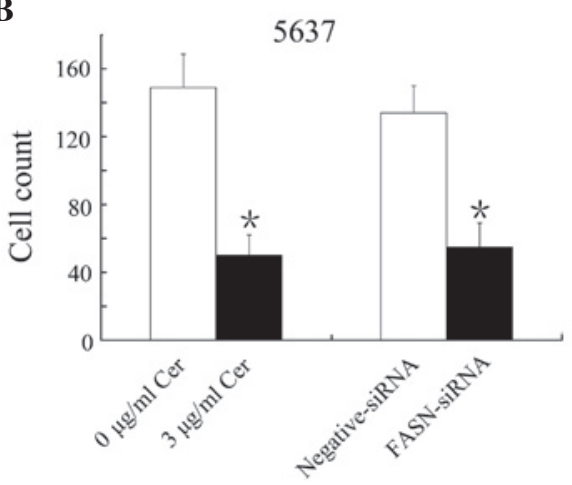

C

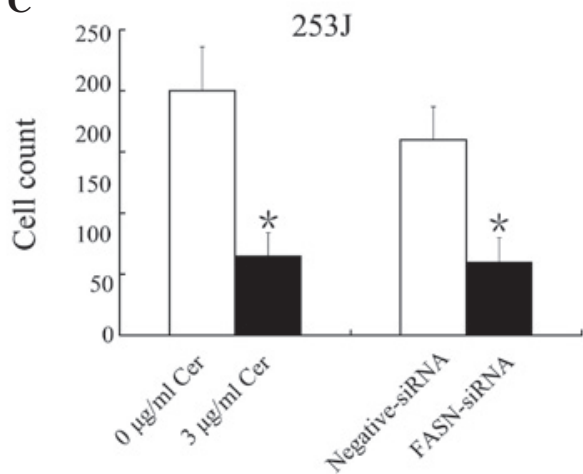

Figure 2. Effects of FASN inhibition by knockdown with FASN-siRNA or the pharmacological inhibitor Cer on the migration of bladder transitional cell carcinoma cell lines. (A) 5637 and 253J cells were treated with $3 \mu \mathrm{g} / \mathrm{ml} \mathrm{Cer} \mathrm{for} 24 \mathrm{~h}$ or transfected with FASN-siRNA for $48 \mathrm{~h}$ and the number of cells transgressed through a membrane was observed by microscopy (magnification, x400). (B and C) Quantification of A showed that FASN-siRNA and Cer obviously suppressed the migration of 5637 and 253J cells. Images are representative of three separate experiments. Values are expressed as the mean \pm standard deviation; "P<0.05. FASN, fatty acid synthase complex; siRNA, small interfering RNA; Cer, cerulenin.

A

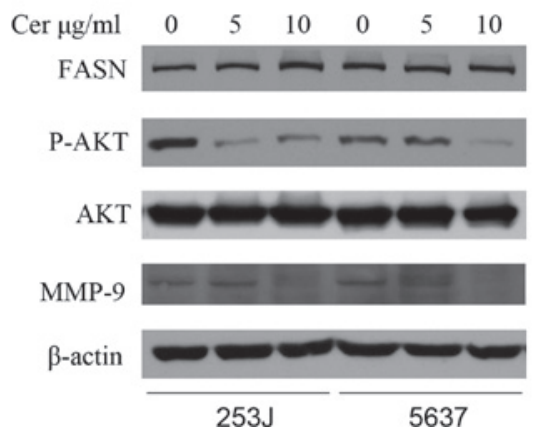

B

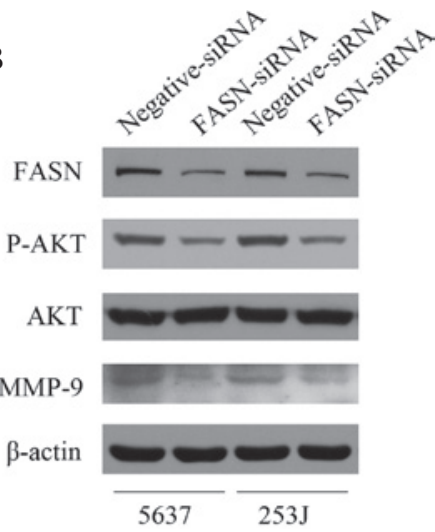

Figure 3. FASN inhibition downregulates AKT and MMP-9 activity in bladder transitional cell carcinoma cell lines. (A) FASN inhibitor Cer (5 or $10 \mu \mathrm{g} / \mathrm{ml}$ for $24 \mathrm{~h}$ ) downregulated p-AKT and MMP-9, while it had no effect on FASN and AKT expression. (B) FASN knockdown by FASN-siRNA downregulated p-AKT and MMP-9. $\beta$-actin served as the internal control. FASN, fatty acid synthase complex; siRNA, small interfering RNA; Cer, cerulenin. MMP, matrix metalloproteinase; p-AKT, phosphorylated AKT.

Transwell assay. Cell migration was assessed using Transwell pates (pore size, $8 \mu \mathrm{m}$; Costar; Corning Inc., Corning, NY, USA). Following transfection with FASN-siRNA for $48 \mathrm{~h}$ or treatment with $3 \mu \mathrm{g} / \mathrm{ml}$ Cer for $24 \mathrm{~h}, 5637$ and 253J cells were washed once with phosphate-buffered saline (PBS). $200 \mathrm{ml}$ serum-free RPMI 1640 medium containing $1 \times 10^{5}$ cells and $3 \mu \mathrm{g} / \mathrm{ml}$ Cer in the Cer treatment group was placed in the upper chamber, and the lower chamber was filled with $500 \mathrm{ml}$ of the same medium. Subsequent to incubation of the plates for $24 \mathrm{~h}$ at $37^{\circ} \mathrm{C}$, cells on the upper side of the filters were removed with cotton-tipped swabs. Following fixing in methanol (Gibco; Thermo Fisher Scientific, Inc.), cells on the lower side of the filter were stained with $0.1 \%$ of crystal violet in PBS (Gibco; Thermo Fisher Scientific, Inc.). The number of migrated cells was quantified in six random high-power microscopic fields per sample (BX50; Olympus Corporation). Transwell assays were performed in triplicate.

Statistical analysis. SPSS 18.0 for Windows (SPSS, Inc., Chicago, IL, USA) was used to analyze all data. Values 
are expressed as the mean \pm standard error of the mean. Comparisons between two groups were performed using Student's t-test and the $\chi^{2}$-test was utilized to assess statistical significance between categorical data. $\mathrm{P}<0.05$ was considered to indicate a statistically significant difference between values.

\section{Results}

siRNA-mediated FASN knockdown. According to a previous study by our group evaluating three different siRNA sequences targeting FASN (11), the most efficient of these siRNAs was used in the present study to knockdown FASN expression in 5637 and 253J cells. To determine the transfection efficiency, 5637 and 253J cells were transfected with FAM-siRNA for $12 \mathrm{~h}$ respectively and FAM fluorescence was detected by fluorescence microscopy (Fig. 1A). The amount of positively transfected cells observed visually to exceed $70 \%$. To assess the knockdown efficiency of FASN-siRNA in 5637 and 253J cells, mRNA and protein expression were detected using RT-qPCR and western blot analysis (Fig. 1B and C). Transfection with FASN-siRNA resulted in a significant reduction of FASN mRNA and protein expression in the BTCC cell lines. These results indicated that FASN-siRNA was successfully transfected into 5637 and 253J cells and efficiently knocked down FASN.

FASN inhibition by Cer and FASN-siRNA suppresses the migratory capacity of BTCC cells. The present study assessed whether inhibition of FASN expression affected the migration of 5637 and 253J cells using a Transwell assay. Treatment with $3 \mu \mathrm{g} / \mathrm{ml}$ Cer for $24 \mathrm{~h}$ or transfection with FASN-siRNA for $48 \mathrm{~h}$ significantly decreased the percentage of 5637 and 253J cells that had transgressed through the Transwell filter (Fig. 2A-C). The results therefore indicated that FASN inhibition suppressed BTCC-cell migration.

FASN inhibition de-activates AKT and reduces MMP-9 expression. To identify the mechanism by which FASN inhibition reduced the migratory capacity of BTCC cells, two key regulatory factors of cell-migration, MMP-9 and AKT, were assessed. MMP-9, an important indicator of tumor migration, has been shown to be involved in the migratory capacity of bladder cancer cells $(13,14)$. Furthermore, the phosphoinositide-3 kinase (PI3K)/AKT pathway is one of the most important signaling pathways and can regulate migration and invasion of bladder cancer cells $(15,16)$. Following transient transfection of 5637 and 253J cells with siRNA for $48 \mathrm{~h}$ or treatment with 5 and $10 \mu \mathrm{g} / \mathrm{ml}$ Cer for $24 \mathrm{~h}$, the protein levels of MMP-9 and p-AKT were found to be reduced (Fig. 3A and B). However, Cer did not affect FASN expression in these cell lines (Fig. 3A).

\section{Discussion}

The natural product Cer is a mycotoxin which was initially developed as an anti-fungal agent and is now widely used as a natural inhibitor of FASN activity $(17,18)$. Furthermore, transfection with siRNA is a commonly used method for silencing the expression of specific genes (19). In the present study, FASN-siRNA and FASN inhibitor Cer were used to inhibit
FASN expression in the 5637 and 253J BTCC cell lines, which led to a decrease in the cells' migratory potential. This was accompanied by decreases in MMP-9 expression and p-AKT levels, indicating that FASN inhibitor Cer and FASN-siRNA reduced the migratory capacity of BTCC cells via suppressing the activation of AKT, leading to a downregulation of MMP-9 expression.

The role of FASN in cancer has become a focus of current research on the discovery of novel diagnostic tools and treatments for cancer. FASN expression and sensitivity to FASN-targeting drugs are directly linked to cell growth, while only being indirectly correlated with transformation, differentiation and senescence in various cancer types, including breast (20), prostate (21), ovarian (22), lung (23) and liver cancer (24). In addition, FASN was also found to be involved in cancer occurrence (25), progression (26), metastasis (27) and chemotherapeutic resistance (28) in various types of cancer. FASN is therefore a metabolic marker of cell proliferation as well as a useful target for future drug development.

Previous studies indicated that FASN is correlated with metastasis in several types of tumor cell $(29,30)$. However, in BTCC cells, the regulatory function of FASN on the migratory capacity and the detailed underlying mechanisms have remained elusive. The present study assessed the effects of FASN inhibition on the expression of MMP-9, which is able to promote cell migration, in human BTCC cells. The observed reduction of MMP-9 expression was likely to be based on the simultaneous suppression of p-AKT. Therefore, inhibition of FASN suppresses the migratory capacity of BTCC cells, at least in part, by de-activating p-AKT. These results indicated that FASN may be a potential target for BTCC therapy.

Cancer metastasis, which is the main cause of mortality in cancer patients, is a complex multi-step process facilitated by several molecular key events. Identifying and targeting various associated genes is the most promising strategy to treat or prevent metastasis $(2,31,32)$. MMPs are a family of zinc-dependent endopeptidases which participate in the proteolytic destruction of basement and extracellular matrix membranes, therefore being essential for tumor invasion and metastasis $(33,34)$. Among them, MMP-9 is one of the most important markers of cancer invasion and metastasis (35). A previous study has shown that MMP-9 is implicated in metastasis of bladder cancer (36). In addition, MMP-9 was shown to be associated with the pathological grade, clinical stage and prognosis of tumors (37-39). Therefore, the present study assessed whether FASN inhibition regulates MMP-9 expression and found that the protein levels of MMP-9 were significantly downregulated.

In recent years, the PI3K/AKT signaling pathway has attracted broad scientific and clinical interest as a cancer drug target $(40,41)$. It is thought that activation of the PI3K/AKT signaling pathway is associated with invasion and metastasis of cancer cells $(42,43)$. The activated form of AKT, p-AKT, has a vital role in cell-cycle progression, the apoptotic program and the migratory capacity of cells via downregulating the expression of caspase 3 and upregulating MMP-9 (44). Therefore, inhibition of the PI3K/AKT pathway is regarded as a potential strategy for the treatment of cancer $(45,46)$. In addition, FASN inhibition was shown to decrease AKT activity, which was, at least in part, based on a reduction of membrane phospholipid production (47). A previous study by our group 
also supported the notion that inhibition of FASN suppresses the AKT pathway (11). However, to date, the effects of FASN on BTCC-cell migration and the possible involvement of the PI3K/AKT pathway or MMPs has remained elusive. The present study, showed that FASN inhibition downregulated MMP-9 expression by targeting the AKT pathway in BTCC cells to reduce cell migration. Thus, the present study not only provided further evidence for FASN inhibition being a promising therapeutic approach to control bladder-cancer invasion and metastasis, but also revealed the underlying mechanism via the PI3K/AKT pathway and MMP-9.

The present study provided further evidence that FASN has an important role in BTCC. FASN inhibition suppressed the migratory capacity of BTCC cells through downregulating MMP-9 expression; furthermore, the PI3K/AKT signaling pathway was indicated to be involved. These findings may enhance the understanding of the underlying mechanisms of the inhibitory effects of FASN knockdown on the migratory capacity of BTCC cells, and suggested that targeting FASN may be a potential therapeutic strategy for BTCC.

\section{References}

1. Shariat SF, Karakiewicz PI, Palapattu GS, Lotan Y, Rogers CG, Amiel GE, Vazina A, Gupta A, Bastian PJ, Sagalowsky AI, et al: Outcomes of radical cystectomy for transitional cell carcinoma of the bladder: A contemporary series from the Bladder Cancer Research Consortium. J Urol 176 : 2414-2422, 2006.

2. Madersbacher S, Hochreiter W, Burkhard F, Thalmann GN, Danuser H, Markwalder R and Studer UE: Radical cystectomy for bladder cancer today-a homogeneous series without neoadjuvant therapy. J Clin Oncol 21: 690-696, 2003.

3. Philips GK, Halabi S, Sanford BL, Bajorin D and Small EJ; Cancer and Leukemia Group B: A phase II trial of cisplatin $(\mathrm{C})$, gemcitabine $(\mathrm{G})$ and gefitinib for advanced urothelial tract carcinoma: Results of Cancer and Leukemia Group B (CALGB) 90102. Ann Oncol 20: 1074-1079, 2009.

4. Wu M, Dickinson SI, Wang X and Zhang J: Expression and function of SIRT6 in muscle invasive urothelial carcinoma of the bladder. Int J Clin Exp Pathol 7: 6504-6513, 2014.

5. Ferlay J, Soerjomataram I, Dikshit R, Eser S, Mathers C, Rebelo M, Parkin DM, Forman D and Bray F: Cancer incidence and mortality worldwide: Sources, methods and major patterns in GLOBOCAN 2012. Int J Cancer 136: E359-E386, 2015.

6. Kuhajda FP, Jenner K, Wood FD, Hennigar RA, Jacobs LB, Dick JD and Pasternack GR: Fatty acid synthesis: A potential selective target for antineoplastic therapy. Proc Natl Acad Sci USA 91: 6379-6383, 1994.

7. Wakil SJ: Fatty acid synthase, a proficient multifunctional enzyme. Biochemistry 28: 4523-4530, 1989.

8. Kuhajda FP: Fatty acid synthase and cancer: New application of an old pathway. Cancer Res 66: 5977-5980, 2006.

9. Orita H, Coulter J, Lemmon C, Tully E, Vadlamudi A, Medghalchi SM, Kuhajda FP and Gabrielson E: Selective inhibition of fatty acid synthase for lung cancer treatment. Clin Cancer Res 13: 7139-7145, 2007.

10. Abdel-Magid AF: Fatty acid synthase (FASN) inhibitors as potential treatment for cancer, obesity, and liver related disorders. ACS Med Chem Lett 6: 838-839, 2015.

11. Jiang B, Li EH, Lu YY, Jiang Q, Cui D, Jing YF and Xia SJ: Inhibition of fatty-acid synthase suppresses P-AKT and induces apoptosis in bladder cancer. Urology 80: 484.e9-e15, 2012.

12. Livak KJ and Schmittgen TD: Analysis of relative gene expression data using real-time quantitative PCR and the 2(-Delta Delta C(T)) method. Methods 25: 402-408, 2001.

13. Saeb-Parsy K, Veerakumarasivam A, Wallard MJ, Thorne N, Kawano Y, Murphy G, Neal DE, Mills IG and Kelly JD: MT1-MMP regulates urothelial cell invasion via transcriptional regulation of Dickkopf-3. Br J Cancer 99: 663-669, 2008.

14. Di Carlo A, Terracciano D, Mariano A and Macchia V: Urinary gelatinase activities (matrix metalloproteinases 2 and 9) in human bladder tumors. Oncol Rep 15: 1321-1326, 2006.
15. Houédé $\mathrm{N}$ and Pourquier P: Targeting the genetic alterations of the PI3K-AKT-mTOR pathway: Its potential use in the treatment of bladder cancers. Pharmacol Ther 145: 1-18, 2015.

16. Chen M, Gu J, Delclos GL, Killary AM, Fan Z, Hildebrandt MA, Chamberlain RM, Grossman HB, Dinney CP and Wu X: Genetic variations of the PI3K-AKT-mTOR pathway and clinical outcome in muscle invasive and metastatic bladder cancer patients. Carcinogenesis 31: 1387-1391, 2010.

17. Cheng G, Palanisamy AP, Evans ZP, Sutter AG, Jin L, Singh I, May H, Schmidt MG and Chavin KD: Cerulenin blockade of fatty acid synthase reverses hepatic steatosis in ob/ob mice. PLoS One 8: e75980, 2013

18. Nomura S, Horiuchi T, Hata T and Omura S: Inhibition of sterol and fatty acid biosyntheses by cerulenin in cell-free systems of yeast. J Antibiot (Tokyo) 25: 365-368, 1972.

19. Valencia-Sanchez MA, Liu J, Hannon GJ and Parker R: Control of translation and mRNA degradation by miRNAs and siRNAs. Genes Dev 20: 515-524, 2006.

20. Bessadóttir M, Skúladóttir EÁ, Gowan S, Eccles S, Ögmundsdóttir S and Ogmundsdóttir HM: Effects of anti-proliferative lichen metabolite, protolichesterinic acid on fatty acid synthase, cell signalling and drug response in breast cancer cells. Phytomedicine 21: 1717-1724, 2014.

21. Sadowski MC, Pouwer RH, Gunter JH, Lubik AA, Quinn RJ and Nelson CC: The fatty acid synthase inhibitor triclosan: Repurposing an anti-microbial agent for targeting prostate cancer. Oncotarget 5: 9362-9381, 2014.

22. Veigel D, Wagner R, Stübiger G, Wuczkowski M, Filipits M, Horvat R, Benhamú B, López-Rodríguez ML, Leisser A, Valent $\mathrm{P}$, et al: Fatty acid synthase is a metabolic marker of cell proliferation rather than malignancy in ovarian cancer and its precursor cells. Int J Cancer 136: 2078-2090, 2015.

23. Jin X, Zhang KJ, Guo X, Myers R, Ye Z, Zhang ZP, Li XF, Yang HS and Xing JL: Fatty acid synthesis pathway genetic variants and clinical outcome of non-small cell lung cancer patients after surgery. Asian Pac J Cancer Prev 15: 7097-7103, 2014.

24. Impheng H, Pongcharoen S, Richert L, Pekthong D and Srisawang P: The selective target of capsaicin on FASN expression and de novo fatty acid synthesis mediated through ROS generation triggers apoptosis in HepG2 cells. PLoS One 9: e107842, 2014.

25. Migita T, Ruiz S, Fornari A, Fiorentino M, Priolo C, Zadra G, Inazuka F, Grisanzio C, Palescandolo E, Shin E, et al: Fatty acid synthase: A metabolic enzyme and candidate oncogene in prostate cancer. J Natl Cancer Inst 101: 519-532, 2009.

26. Menendez JA, Vellon L, Mehmi I, Oza BP, Ropero S, Colomer R and Lupu R: Inhibition of fatty acid synthase (FAS) suppresses HER2/neu (erbB-2) oncogene overexpression in cancer cells. Proc Natl Acad Sci USA 101: 10715-10720, 2004.

27. Murata S, Yanagisawa K, Fukunaga K, Oda T, Kobayashi A, Sasaki R and Ohkohchi N: Fatty acid synthase inhibitor cerulenin suppresses liver metastasis of colon cancer in mice. Cancer Sci 101: 1861-1865, 2010.

28. Zeng L, Biernacka KM, Holly JM, Jarrett C, Morrison AA, Morgan A, Winters ZE, Foulstone EJ, Shield JP and Perks CM: Hyperglycaemia confers resistance to chemotherapy on breast cancer cells: The role of fatty acid synthase. Endocr Relat Cancer 17: 539-551, 2010.

29. Murata S, Yanagisawa K, Fukunaga K, Oda T, Kobayashi A, Sasaki R and Ohkohchi N: Fatty acid synthase inhibitor cerulenin suppresses liver metastasis of colon cancer in mice. Cancer Sci 101: 1861-1865, 2010.

30. Wellberg EA, Rudolph MC, Lewis AS, Padilla-Just N, Jedlicka P and Anderson SM: Modulation of tumor fatty acids, through overexpression or loss of thyroid hormone responsive protein spot 14, is associated with altered growth and metastasis. Breast Cancer Res 16: 481, 2014.

31. Valastyan S and Weinberg RA: Tumor metastasis: Molecular insights and evolving paradigms. Cell 147: 275-292, 2011.

32. Hanahan D and Weinberg RA: Hallmarks of cancer: The next generation. Cell 144: 646-674, 2011.

33. Nabeshima K, Inoue T, Shimao Y and Sameshima T: Matrix metalloproteinases in tumor invasion: Role for cell migration. Pathol Int 52: 255-264, 2002.

34. Overall CM and Kleifeld O: Tumour microenvironment-opinion: Validating matrix metalloproteinases as drug targets and anti-targets for cancer therapy. Nat Rev Cancer 6: 227-239, 2006.

35. Egeblad $M$ and Z Werb: New functions for the matrix metalloproteinases in cancer progression. Nat Rev Cancer 2: 161-174, 2002. 
36. Kumar B, Koul S, Petersen J, Khandrika L, Hwa JS, Meacham RB, Wilson $S$ and Koul HK: p38 mitogen-activated protein kinase-driven MAPKAPK2 regulates invasion of bladder cancer by modulation of MMP-2 and MMP-9 activity. Cancer Res 70: 832-841, 2010.

37. Offersen BV,Knap MM,Horsman MR, Verheijen J, Hanemaaijer R and Overgaard J: Matrix metalloproteinase-9 measured in urine from bladder cancer patients is an independent prognostic marker of poor survival. Acta Oncol 49: 1283-1287, 2010.

38. Donmez G, Sullu Y, Baris S, Yildiz L, Aydin O, Karagoz F and Kandemir B: Vascular endothelial grow th factor (VEGF), matrix metalloproteinase-9 (MMP-9) and thrombospondin-1 (TSP-1) expression in urothelial carcinomas. Pathol Res Pract 205: 854-857, 2009.

39. Vasala K, Pääkko P and Turpeenniemi-Hujanen T: Matrix metalloproteinase-9 (MMP-9) immunoreactive protein in urinary bladder cancer: A marker of favorable prognosis. Anticancer Res 28(3B): 1757-1761, 2008

40. Martelli AM, Tazzari PL, Evangelisti C, Chiarini F, Blalock WL, Billi AM, Manzoli L, McCubrey JA and Cocco L: Targeting the phosphatidylinositol 3-kinase/Akt/mammalian target of rapamycin module for acute myelogenous leukemia therapy: From bench to bedside. Curr Med Chem 14: 2009-2023, 2007.

41. Engelman JA, Luo J and Cantley LC: The evolution of phosphatidylinositol 3-kinases as regulators of growth and metabolism. Nat Rev Genet 7: 606-619, 2006.
42. Carneiro BA, Meeks JJ, Kuzel TM, Scaranti M, Abdulkadir SA and Giles FJ: Emerging therapeutic targets in bladder cancer. Cancer Treat Rev 41: 170-178, 2015.

43. Paplomata E and O'Regan R: The PI3K/AKT/mTOR pathway in breast cancer: Targets, trials and biomarkers: 154-166, 2014.

44. Chen H, Yang X, Feng Z, Tang R, Ren F, Wei K and Chen G: Prognostic value of caspase- 3 expression in cancers of digestive tract: A meta-analysis and systematic review. Int J Clin Exp Med 8: 10225-10234, 2015.

45. Wu S, Ju GQ, Du T, Zhu YJ and Liu GH: Microvesicles derived from human umbilical cord Wharton's jelly mesenchymal stem cells attenuate bladder tumor cell growth in vitro and in vivo. PLoS One 8: e61366, 2013.

46. Jeong JW, Jin CY, Park C, Han MH, Kim GY, Moon SK, Kim CG, Jeong YK, Kim WJ, Lee JD and Choi YH: Inhibition of migration and invasion of $\mathrm{LNCaP}$ human prostate carcinoma cells by cordycepin through inactivation of Akt. Int J Oncol 40: 1697-1704, 2012.

47. Swinnen JV, Van Veldhoven PP, Timmermans L, De Schrijver E, Brusselmans K, Vanderhoydonc F, Van de Sande T, Heemers H, Heyns W and Verhoeven G: Fatty acid synthase drives the synthesis of phospholipids partitioning into detergent-resistant membrane microdomains. Biochem Biophys Res Commun 302: 898-903, 2003 